5. Stackebrandt E, Koch C, Gvozdiak O, Schumann P. Taxonomic dissection of the genus Micrococcus: Kocuria gen. nov., Nesterenkonia gen. nov., Kytococcus gen. nov., Dermacoccus gen. nov., and Micrococcus Cohn 1872 gen. emend. Int J Syst Bacteriol 1995;45:682-92.

6. Ellinghaus $\mathrm{P}$, Badehorn $\mathrm{D}$, Blümer R, Becker K, Seedorf U. Increased efficiency of arbitrarily primed PCR by prolonged ramp times. Biotechniques 1999;26:626-8.

7. Becker K, Schumann P, Wüllenweber J, Schulte M, Weil HP, Stackebrandt E, et al. Kytococcus schroeteri sp. nov., a novel gram-positive actinobacterium isolated from a human clinical source. Int J Syst Evol Microbiol 2002;52:1609-14.

8. Rhoden DL, Miller JM. Four-year prospective study of STAPH-IDENT system and conventional method for reference identification of Staphylococcus, Stomatococcus, and Micrococcus spp. J Clin Microbiol 1995;33:96-8.

9. Peces R, Gago E, Tejada F, Laures AS, Alvarez-Grande J. Relapsing bacteraemia due to Micrococcus luteus in a haemodialysis patient with a Perm-Cath catheter. Nephrol Dial Transplant 1997;12:2428-9.

10. von Eiff C, Herrmann M, Peters G. Antimicrobial susceptibilities of Stomatococcus mucilaginosus and of Micrococcus spp. Antimicrob Agents Chemother 1995;39:268-70.

Address for correspondence: Karsten Becker, University of Münster, Institute of Medical Microbiology, Domagkstr. 10, 48149 Münster, Germany; fax: (49) 251 83-55350; email: kbecker@uni-muenster.de

\section{When Is a Reservoir Not a Reservoir?}

To the Editor: Some $80 \%$ of parasitic infections of humans are zoonoses (1). These infections are caused by multihost parasites for which the reservoir of infection depends on hosts other than Homo sapiens. But what is a reservoir of infection?

Haydon et al. (2) proposed a new series of definitions in connection with multihost pathogens, in which a target host is the host of interest in a particular context. The reservoir of infection included, for these authors, all hosts, whether incidental or not, that are epidemiologically connected to (i.e., contribute to transmission to) the target host.

The availability of three terms reservoir, reservoir of infection, and reservoir host - frequently used interchangeably, leads to confusion. This confusion is, in part, what prompted me (3) to slightly redefine a reservoir (of infection) as an ecologic system in which an infectious agent survives indefinitely. Such a system includes all the component host populations, including that of any intermediate host or vector, in the context of any environmental component, and any quantitative requisite such as critical community size, which is required to maintain the agent indefinitely.

Vertebrate hosts that form an essential part of the system are reservoir hosts, though whether a whale or a fish is the reservoir host of Anisakis species can be a matter of debate. A host that becomes infected, but is not required for the maintenance of the population of a pathogen, can usefully be called an incidental host. (Accidental host is frequently used, but this is arguably a teleological term and therefore undesirable.) For incidental hosts that transmit pathogens from a reservoir to another incidental host, analogous to a pipe leading from a water reservoir, Garnham (4) coined the useful term "liaison host."

Haydon et al. dismiss my definition on two grounds. First, I exclude liaison hosts from the reservoir. This distinction is valid and could be argued either way, but I suggest that the pipes leading from a reservoir do not form part of the reservoir and that it is both conceptually and practically important to distinguish liaison hosts from reservoir hosts. The second objection is that many pathogens depend on the presence of several host species, at any given stage in the life history, for their maintenance. This concept is clearly considered in my article: together, such hosts collectively constitute part of the reservoir system, though no single one may be the reservoir host in its own right.

In good scientific English, each term should have a precise definition, and synonyms should be avoided. The Oxford English Dictionary (OED) (5) definitions of reservoir generally refer to a place or container used for the collection and storage of water, other fluids, or even solid material.

The OED definition of reservoir as a medical term is. "A population which is chronically infested with the causative agent of a disease and can infect other populations." While one might argue with the terms chronically, infested, and the infection of populations, this definition captures the usual sense in which reservoir host is used.

The quotations given in OED are more helpful. The earliest one given for reservoir in a medical context is from 1937, "For the continuous existence of a disease there must be some reservoir of infection... The most important reservoirs of infection are human or animal cases or carriers. Plants may be the reservoir of infection in some of the mycoses." However, according to OED, the compound term "reservoir host" was used earlier, in 1913, "The monkey is most probably the normal reservoir host [for Physaloptera mordens]."

The main conceptual difference between the proposal of Haydon et al. and my own is that mine is more generalized: for a given pathogen in a given place, there is a single reservoir. The proposal of Haydon et al. is more limited to practical considerations: the reservoir for one target host may not be the same as that for another target host in the same place.

The most important contribution of these two publications is that they raise an issue that has confused the literature for many years. Parasitologists, virologists, and bacteriologists should agree on a consensus set 
of terms for the ecologic description of multihost systems. When we all agree on what we are talking about, we will understand each other better.

\section{R.W. Ashford*}

*Liverpool School of Tropical Medicine, Liverpool, United Kingdom

\section{References}

1. Ashford RW, Crewe W. The parasites of Homo sapiens: an annotated checklist of the protozoa, helminths and arthropods for which we are home. London: Taylor and Francis; 2003.

2. Haydon DT, Cleaveland S, Taylor LH, Laurenson MK. Identifying reservoirs of infection: a conceptual and practical challenge. Emerg Infect Dis 2002;8:1468-73.

3. Ashford RW. What it takes to be a reservoir host. Belgian Journal of Zoology 1997;127(Supp11):85-90.

4. Garnham PCC. Progress in parasitology. London: Athlone Press; 1971.

5. OED online. Oxford: Oxford University Press 2003. Available from: URL: http://dictionary.oed.com

Address for correspondence: R.W. Ashford, Liverpool School of Tropical Medicine, Liverpool L3 5QA, UK; email address: ashford@liv.ac.uk

\section{Invasive Mycobacterium marinum Infections}

To the Editor: Mycobacterium marinum infections, commonly known as fish tank granuloma, produce nodular or ulcerating skin lesions on the extremities of healthy hosts. Delay of diagnosis is common, and invasion into deeper structures such as synovia, bursae, and bone occurs in approximately one third of reported casepatients (1).

A 49-year-old man with diabetes, who had received a kidney transplant from a living relative 8 years previously, sought treatment after 5 months of worsening swelling and tenderness of the left elbow. Of note, he had injured his left ring finger while cleaning barnacles from a piling 5 years previously and had contracted a secondary infection that never completely healed despite three courses of antimicrobial drugs and surgical debridement. Physical examination showed marked swelling, tenderness, and warmth of the left elbow, as well as of the left ring finger, which was erythematous. Sterile aspiration of the olecranon bursa showed 7,500 leukocytes (62\% lymphocytes) and 141,000 erythrocytes. Results of Gram stain and routine cultures were negative. Magnetic resonance imaging of the left arm showed soft tissue edema of the olecranon bursa and the left fourth flexor digitorum longus tendon, and no osteomyelitis. Three weeks later, olecranon bursa aspirate fluid cultures incubated on chocolate agar and 7H11 plates at $31^{\circ} \mathrm{C}$, as well as on algae slant, and mycobacterial growth indicator tubes incubated at $37^{\circ} \mathrm{C}$ grew M.ycobacterium marinum. The isolate was susceptible to most agents but showed intermediate susceptiblity to ciprofloxacin (MIC $2 \mu \mathrm{g} / \mathrm{mL}$ ) and was resistant to ampicillin/clavulanate and erythromycin (MIC $8 \mu \mathrm{g} / \mathrm{mL}$ and 32 $\mu \mathrm{g} / \mathrm{mL}$, respectively). A treatment regimen of rifampin and ethambutol was begun, and the patient showed a dramatic improvement in the ensuing several weeks. The patient has completed 9 of 11.4 planned months of therapy and continues to do well, with frequent office visits.

Case reports from English language MEDLINE articles since 1966 under the subject heading Mycobacterium marinum were cross-referenced with articles containing the following text words: disseminated, osteomyelitis, arthritis, synovitis, and bursitis. Ten case reports were identified, and a hand search through pertinent articles' references yielded 13 additional reports. A total of 35 cases of invasive $M$. marinum disease were then reviewed, according to patient age and sex, symptoms, source of infection, immune impairment, time to diagnosis, and type as well as duration of therapy (2-24) (see online Table at http://www.cdc.gov/ ncidod/EID/vol9no11/03-0192.htm\# table).

Most cases occurred in previously healthy adults. The average age was 43 years; $24(69 \%)$ were men; 21 (60\%) had tenosynovitis; $6(17 \%)$ had septic arthritis; and 13 (37\%) had osteomyelitis. In three patients $(9 \%)$, either a bone marrow or blood culture positive for $M$. marinum was obtained; all three patients showed marked systemic immunocompromise. Multiple skin lesions were seen in $23 \%$ of cases; half of these patients showed clear evidence of deeper infection. Some patients had more than one manifestation of invasive disease. Immunologic impairment was a frequent component of invasive M. marinum infections: $14(40 \%)$ of case-patients received a steroid injection at the site of infection, and 9 $(26 \%)$ were receiving systemic steroids for various indications. An additional 4 (11\%) case-patients were in an immunocompromised state from other sources such as chemotherapy or AIDS. Delayed diagnosis was also a prominent finding: The average time to diagnosis was 17 months from symptom onset. The treatment course was prolonged and aggressive: The average treatment duration was 11.4 months in the 20 reports in which a definitive duration was given. Surgery was undertaken in $69 \%$ of the cases. The treatment regimen used varied considerably, although $30(88 \%)$ of the 34 patients who took antimycobacterial medications received combination therapy. Rifampin (76\%) and ethambutol $(68 \%)$ were the predominant agents.

While M. marinum infections usually arise from aquatic trauma in healthy hosts, delayed diagnosis and immune suppression contribute to the pathogenesis of invasive infection. Tenosynovitis is the most common 